\title{
Efecto de diferentes protocolos de aplicación de hierro sobre variables hematológicas en lechones
}

\author{
Anchapanta, R.E.; Quisirumbay, J.R.; Naranjo, M.A. \\ Facultad de Medicina Veterinaria y Zootecnia, Universidad \\ Central del Ecuador, Quito. E-mail: jrquisirumbay@uce.edu.ec
}

\begin{abstract}
Resumen
Anchapanta, R.E.; Quisirumbay, J.R.; Naranjo, M.A.: Efecto de diferentes protocolos de aplicación de hierro sobre variables hematológicas en lechones. Rev. Vet. 31: 2, 137-141, 2020. El objetivo de esta investigación fue evaluar el efecto de diferentes protocolos de administración de hierro en la prevención de anemia en lechones, a través de variables hematológicas como cantidad de hematíes (RBC), hemoglobina (HB), hematocrito (HT0), índices eritrocitarios (VCM, HCM, CHCM), ferritina (FT) y transferrina (TF). El estudio se realizó en el Centro Experimental Uyumbicho, Pichincha (Ecuador). Se utilizó un total de 27 lechones (Landrace $\mathrm{x}$ Yorkshire $\mathrm{x}$ Pietrain) distribuidos en un diseño completamente aleatorizado en tres tratamientos (T). En T1 se aplicó hierro-dextrano $(100 \mathrm{mg} / \mathrm{ml})$, a una única dosis de $2 \mathrm{ml}$ al tercer día de edad; T2 hierrodextrano (100 mg/ml), $1 \mathrm{ml}$ a los días 3 y 7 de edad; y T3 hierro dextrano $(200 \mathrm{mg} / \mathrm{ml}), 1 \mathrm{ml} \mathrm{al}$ día 3 de edad. Adicionalmente se evaluó el peso vivo a los 28 días de edad (destete). Las muestras de sangre fueron tomadas 4 días después de la aplicación. Se encontraron diferencias $(p<0,05)$ entre los tratamientos para las variables RBC, HB y HTO, presentando valores más altos en los lechones del grupo T2. Los índices eritrocitarios, así como FT y TF, no presentaron diferencias $(p>0,05)$. Respecto al peso vivo no hubo diferencias entre los grupos evaluados $(p>0,05)$. Se concluye que la aplicación de dos dosis de hierro-dextrano ( $100 \mathrm{mg} / \mathrm{dl}$ a los 3 y 7 días de edad), fue más efectiva para prevenir la anemia, como lo reflejan los valores de hematíes, hemoglobina y hematocrito. El peso vivo del lechón no se vio favorecido por ninguno de los tratamientos aplicados.
\end{abstract}

Palabras claves: lechones, anemia, hierro-dextrano, variables hematológicas.

\begin{abstract}
Anchapanta, R.E.; Quisirumbay, J.R.; Naranjo, M.A.: Effect of different iron application protocols on hematological variables in piglets. Rev. Vet. 31: 2, 137-141, 2020. The objective of this research work was to evaluate the effect of different iron administration protocols in the prevention of anemia in piglets through hematological variables such as red blood cell count (RBC), hemoglobin (HB), hematocrit (HTO), erythrocyte indexes (VCM, CHM, CHCM), ferritin (FT) and transferrin (TF). The study was conducted at the Uyumbicho Experimental Center, Pichincha (Ecuador), using a total of 27 piglets (Landrace $\mathrm{x}$ Yorkshire $\mathrm{x}$ Pietrain) distributed in a completely randomized design in three treatments $(\mathrm{T})$. Tl was applied iron dextran (100 mg/ $\mathrm{ml})$, at a single dose of $2 \mathrm{ml}$ on the third day of age; T2 iron dextran $(100 \mathrm{mg} / \mathrm{ml}), 1 \mathrm{ml}$ at day 3 and 7 of age; T3 iron dextran $(200 \mathrm{mg} / \mathrm{ml}), 1 \mathrm{ml}$ at day 3 of age. Additionally, the body weight was evaluated at 28 days of age (weaning). Blood samples were taken 4 days after application. Difference $(\mathrm{p}<0.05$ ) was found between the treatments for the variables RBC, HB and HTO, presenting higher values in piglets of the T2 group. Erythrocy te index, FT and TF, showed no difference $(p>0.05)$. Regarding body weight, there was no difference between the groups evaluated $(p>0.05)$. From this work it is concluded that the application of two doses of iron dextran $(100 \mathrm{mg} / \mathrm{dl})$ at days 3 and 7 of age, was more effective in preventing anemia as reflected in the values of red blood cells, hemoglobin and hematocrit. The body weight of the piglet was not favored by any of the treatments applied.
\end{abstract}

Key words: piglets, anemia, iron dextran, hematological variables

\section{INTRODUCCIÓN}

En lechones, la mortalidad pre-destete causa una importante reducción en la productividad de las granjas porcinas. Está íntimamente ligada al manejo del lechón en la primera etapa de su desarrollo. En este período, una de las causas de la mortalidad es el desarrollo de anemia nutricional, ocasionada por la deficiencia de hierro (Fe), afectando el crecimiento de los lechones ${ }^{19}$. 
El Fe es requerido como un componente de la hemoglobina en los eritrocitos. Es también encontrado en el músculo como mioglobina, en el suero como transferrina, en la placenta como uteroferrina, en la leche como lactoferrina y en el hígado como ferritina y hemosiderina ${ }^{7}$. Además, tiene un importante rol en el organismo como componente de varias enzimas metabólicas ${ }^{13}$

La deficiencia de Fe es común en mamíferos y severa en lechones ${ }^{23}$. Estos últimos nacen con aproximadamente $50 \mathrm{mg}$ de $\mathrm{Fe}$, la mayor parte del cual se presenta como hemoglobina ${ }^{29}$. Un alto nivel de Fe en la alimentación de las cerdas durante la etapa final de la gestación, o mediante la administración parenteral de hierro-dextrano en cerdas durante la gestación, no aumenta sustancialmente la transferencia placentaria de Fe hacia los fetos ${ }^{7}$.

El lechón recién nacido tiene que retener entre $7 \mathrm{y}$ $16 \mathrm{mg}$ de Fe al día, o $21 \mathrm{mg}$ de Fe por kg de aumento de peso corporal, para mantener niveles adecuados de hemoglobina y almacenar $\mathrm{Fe}^{4,29}$. La leche de la cerda contiene un promedio de solamente $1 \mathrm{mg}$ de Fe por litro, por lo tanto, los lechones que únicamente se alimentan a partir de leche desarrollan anemia ${ }^{29}$.

Las líneas genéticas modernas tienen tasas de crecimiento más rápidas, con mayores acumulaciones de tejido magro, lo cual supone un mayor volumen de sangre corporal ${ }^{11}$. La única fuente de hierro a disposición del lechón son las reservas hepáticas al nacimiento y la escasa concentración existente en la leche materna. En ambos casos, no son suficientes para cubrir los requerimientos del lechón ${ }^{30}$

Esta deficiencia genera disminución en la ganancia de peso diaria y una reducción del consumo de alimento, que deterioran la eficiencia alimentaria y disminuyen el crecimiento de los animales ${ }^{9}$.

Una práctica habitual es el uso de hierro-dextrano, el cual es comercializado en diferentes concentraciones para prevenir esta deficiencia. El tiempo, dosis y número de inyecciones de hierro-dextrano son altamente variables en la industria porcina. Algunas granjas usan una simple inyección de $200 \mathrm{mg}$ de hierro-dextrano, mientras que otras usan entre 100 y $150 \mathrm{mg}$. Algunas suministran $150 \mathrm{mg}$ al día siguiente al nacimiento y subsecuentemente $150 \mathrm{mg}$ cuando los lechones tienen entre 5 a 7 días de edad.

Así, hay una ligera consistencia en la administración de hierro-dextrano en lechones. El fundamento para la existencia de varios esquemas de inyección de $\mathrm{Fe}$, a menudo están basados en la conveniencia de administración y costo, con limitada consideración del desarrollo posible de anemia ${ }^{1}$.

Pocos veterinarios evalúan la concentración de hemoglobina en lechones como una prueba diagnóstica de rutina. Recientemente se encontró que el estatus de hemoglobina fue asociado con ganancias de peso postdestete ${ }^{3} \mathrm{y}$ que camadas numerosas presentan un mayor riesgo para el desarrollo de anemia por deficiencia de $\mathrm{Fe}$ en comparación con camadas pequeñas ${ }^{18}$.
El objetivo del presente estudio fue evaluar el efecto de tres protocolos de administración de hierro-dextrano vía intramuscular, sobre variables hematológicas tales como concentración de hemoglobina, hematocrito, índices eritrocitarios, ferritina y transferrina.

\section{MATERIAL Y MÉTODOS}

El estudio se realizó en la unidad de producción porcina del Centro Experimental Uyumbicho, perteneciente a la Facultad de Medicina Veterinaria y Zootecnia de la Universidad Central del Ecuador, localizada en la provincia de Pichincha, Ecuador.

Se utilizó un total de 27 lechones (Landrace x Yorkshire $\mathrm{x}$ Pietrain), los cuales fueron alojados en corrales con pisos de cemento, junto a sus madres, donde se les permitió lactar todo el tiempo y se les of reció alimento comercial a partir del séptimo día de vida.

Los cerdos fueron distribuidos de manera aleatoria en 3 tratamientos bajo un diseño completamente al azar $(\mathrm{n}=9)$. Tratamiento 1 (Tl): se utilizó hierro-dextrano $(100 \mathrm{mg} / \mathrm{ml}) 2 \mathrm{ml}$ al tercer día posterior al nacimiento; Tratamiento 2 (T2): hierro-dextrano $(100 \mathrm{mg} / \mathrm{ml}) 1 \mathrm{ml}$ a los días 3 y 7 de edad; y Tratamiento 3 (T3): hierrodextrano $(200 \mathrm{mg} / \mathrm{ml}) 1 \mathrm{ml}$ al día 3 de edad.

Se extrajeron muestras de sangre $(6 \mathrm{ml})$ mediante punción de la vena cava anterior, a los 4 días después de la aplicación de los tratamientos. La sangre se distribuyó en dos tubos de ensayo, uno de ellos con anticoagulante (EDTA) para el análisis de glóbulos rojos (RBC), concentración de hemoglobina (Hb), hematocrito (Hto) e índices eritrocitarios, valores que fueron obtenidos en analizador hematológico Mindray BC-2800.

En el segundo tubo (sin anticoagulante), el suero se utilizó para evaluar ferritina (por electro-quimioluminiscencia, equipo Elecsys 2010 Modular Analitics E170 de la marca Cobas) y transferrina (método inmuno-turbidimétrico empleando un analizador Roche/Hitachi Cobas c311).

Con los valores obtenidos se realizó análisis de varianza (ANOVA) y en caso de existir diferencias significativas se utilizó la prueba de Tukey para comparación de medias entre tratamientos, utilizando el programa estadístico R-studio.

\section{RESULTADOS}

En la Tabla 1 se observan los promedios de las variables evaluadas entre los tres tratamientos de hierrodextrano después de la aplicación de Fe. En comparación con los procedimientos 1 y 3 , los lechones del tratamiento 2 ostentaron mayor $\mathrm{RBC}(\mathrm{p}<0,0042)$, concentración de $\mathrm{Hb}(\mathrm{p}<0,0012)$ y Hto $(\mathrm{p}<0,0025)$, cuatro días después de la administración de hierro-dextrano.

Con relación a los índices eritrocitarios, ferritina y transferrina, no hubo diferencias significativas $(\mathrm{p}<0,05)$ cuatro días después de la administración de hierro-dextrano. En la Tabla 2 se presentan los pesos semanales de los animales hasta el destete. Se obser- 
Tabla 1. Variables hematológicas en lechones tras la aplicación de diferentes protocolos de hierro.

\begin{tabular}{lcccccc}
\hline var & T1 & T2 & T3 & rangos & PV & sig \\
\hline RBC & $4,2 \pm 0,5 \mathrm{~b}$ & $5,4 \pm 0,8 \mathrm{a}$ & $4,4 \pm 0,7 \mathrm{~b}$ & $5-8^{6}$ & 0,0042 & $\mathrm{DS}$ \\
$\mathrm{Hb}$ & $10,1 \pm 0,6 \mathrm{~b}$ & $12,7 \pm 1,7 \mathrm{a}$ & $10,4 \pm 1,4 \mathrm{~b}$ & $9-14^{6}$ & 0,0012 & $\mathrm{DS}$ \\
Hto & $33,6 \pm 2,6 \mathrm{~b}$ & $40,5 \pm 3,7 \mathrm{a}$ & $34,7 \pm 4,6 \mathrm{~b}$ & $32-50^{10}$ & 0,0025 & $\mathrm{DS}$ \\
VCM & $78,8 \pm 5,6$ & $76,4 \pm 7,1$ & $79,1 \pm 5,5$ & $42-62^{6}$ & 0,41 & $\mathrm{DN}$ \\
$\mathrm{HCM}$ & $23,8 \pm 1,3$ & $23,1 \pm 1,5$ & $23,7 \pm 1,6$ & $14-21^{6}$ & 0,44 & $\mathrm{DN}$ \\
$\mathrm{CHCM}$ & $30,2 \pm 1,1$ & $30,4 \pm 1,5$ & $30 \pm 0,6$ & $32-36^{6}$ & 0,72 & $\mathrm{DN}$ \\
$\mathrm{FT}$ & $50,8 \pm 8,5$ & $68,3 \pm 29,1$ & $55 \pm 14,8$ & $11-45^{22}$ & 0,21 & $\mathrm{DN}$ \\
TF & $0,49 \pm 0,1$ & $0,52 \pm 0,1$ & $0,49 \pm 0.1$ & $1,5-2^{27}$ & 0,57 & $\mathrm{DN}$ \\
\hline
\end{tabular}

var: variables; T1-T2-T3: tratamientos $(\mathrm{X} \pm \mathrm{SD})$; $\mathrm{RBC}$ (x106/ul): número total de eritrocitos; $\mathrm{Hb}$ (g/dl): hemoglobina; Hto (\%): hematocrito; VCM (fl): volumen corpuscular medio; HCM (pg): hemoglobina corpuscular media (\%); $\mathrm{CHC}$ ( $\mathrm{g} / \mathrm{dl}$ ): concentración de hemoglobina corpuscular media; FT (ng/ml): ferritina; TF (g/L): transferrina. T1, T2, T3: tratamientos. PV: pvalue. sig: significación (DS: diferencia significativa, DN: no significativa). Las comparaciones entre tratamientos se efectúan en la misma fila, literales similares representan igualdad entre grupos.

Tabla 2. Pesos de los animales desde el inicio del estudio hasta el destete día 28 .

\begin{tabular}{lccccc}
\hline variables & semana & tratam.1 & tratam. 2 & tratam.3 & p-value \\
\hline peso $(\mathrm{kg})$ & 1 & $2,75 \pm 0,42$ & $2,70 \pm 0,47$ & $2,70 \pm 0,52$ & 0,369 \\
peso $(\mathrm{kg})$ & 2 & $4,21 \pm 0,76$ & $4,17 \pm 0,75$ & $4,27 \pm 0,94$ & 0,531 \\
peso $(\mathrm{kg})$ & 3 & $5,63 \pm 1,15$ & $5,54 \pm 1,09$ & $5,68 \pm 1,15$ & 0,473 \\
peso $(\mathrm{kg})$ & 4 & $6,98 \pm 1,62$ & $7,07 \pm 1,53$ & $6,38 \pm 1,00$ & 0,347 \\
\hline
\end{tabular}

va que no hubo diferencias estadísticas significativas $(p>0,05)$ entre los pesos obtenidos en los tres tratamientos al término del estudio.

\section{DISCUSIÓN}

La deficiencia de hierro es un serio problema en la producción porcina, debido a que los lechones tienen limitadas reservas, imposibilitando cubrir los requerimientos de este micronutriente. Sin aplicación de fuentes de hierro los lechones desarrollan anemia dentro de 10 a 14 días después del nacimiento $5,8,17,25,28$.

En la evaluación realizada 4 días después de la aplicación de hierro-dextrano se pudo observar que los tres tratamientos utilizados previnieron eficientemente el deterioro del estado hematológico de los lechones recién nacidos.

Sin embargo, el tratamiento 2 (donde se administró $100 \mathrm{mg}$ de hierro a los días 3 y 7 de edad), presentó diferencia significativa $(p<0,05)$ en los valores de $R B C$, $\mathrm{Hb}$ y Hto en comparación con los tratamientos 1 y 3 . El tratamiento 2 presentó un valor $\mathrm{RBC}$ de $5,4 \times 10^{6} / \mathrm{ul}$, superior a T1 y T3. Este valor concuerda con lo reportado por un investigador ${ }^{6}$ que halló para dicha variable un rango entre 5,3 a $8 \times 10^{6} / \mathrm{ul}$.

El valor del Hto fue de $40,54 \%$ en el T2 (superior a T1 y T3, p<0,05), encontrándose dentro de los valores reportados por investigadores que señalan que un he- matocrito de 32 a $50 \%$ es el óptimo para lechones de esta edad ${ }^{10}$.

Con relación a la $\mathrm{Hb}$, varios autores asumen que esta variable es la más utilizada en la industria porcina para evaluar la deficiencia de hierro o anemia. En nuestro estudio $\mathrm{T} 2$ mostró el valor más alto de hemoglobina $(12,74 \mathrm{~g} / \mathrm{dl})$ en comparación con los otros tratamientos $(p<0,05)$. El valor de $\mathrm{T} 2$ se encuentra dentro del rango reportado por varios investigadores 14,16 , ${ }^{25}$, quienes señalaron una concentración de $\mathrm{Hb}$ de $10 \mathrm{~g} / \mathrm{dl}$ o superior, indicando un adecuado contenido de hierro en el organismo y manifestando que valores inferiores pueden comprometer la salud de los animales.

Con resultados similares, algunos investigadores encontraron que una segunda aplicación de hierro (100 mg) a los 10 días de edad, provocó concentraciones altas de hemoglobina a los 14 días posteriores al destete ${ }^{11}$.

Otros recomiendan calcular tres variables como indicadoras tempranas de una deficiencia de hierro: volumen corpuscular medio (VCM), hemoglobina corpuscular media (HCM) y concentración de hemoglobina corpuscular media $(\mathrm{CHCM})^{3,24}$. Las tres variables disminuyen en estados de anemia, sin embargo -en el presente estudio- los valores de los índices eritrocitarios se hallaron dentro de niveles normales, sin existir diferencia entre los tratamientos $(p>0,05)$.

Para evaluar el estado del hierro también se examinaron los niveles séricos de ferritina y transferrina, los cuales no registraron diferencias significativas $(p>0,05)$ entre los diferentes tratamientos. Tal hallazgo resultó similar al reportado por investigadores quienes encontraron que con la aplicación de hierro-dextrano, tanto la ferritina como la transferrina se mantuvieron estables y que en los cerdos que no recibieron hierrodextrano se observó una marcada disminución de estos depósitos, señalando que ellos van disminuyendo con el crecimiento de los lechones ${ }^{20,27}$.

Tales resultados fueron similares a los de investigadores que probaron varios protocolos de administración suplementaria de hierro que comprendían protocolos con dosis únicas y protocolos con dosis divididas, obteniendo los mejores resultados en los protocolos con dosis divididas, mejorando los valores hematológicos ${ }^{15,23}$

Otros estudios confirmaron que la administración de grandes cantidades de hierro pueden generar efectos tóxicos al inducir la expresión de hepcidina, la misma que puede disminuir la biodisponibilidad de hierro suplementario, por lo cual recomendaron el uso de protocolos con suplementación dividida ${ }^{20,26}$. Respecto a la evaluación del peso vivo, se encontró que en animales 
no tratados con hierro, el peso disminuyó gradualmente a partir de la tercera semana de edad, obteniendo pesos menores que los animales tratados con hierrodextrano ${ }^{8}$.

El presente estudio mostró la inexistencia de efectos de los 3 tratamientos investigados sobre el peso vivo durante el período de lactancia. Resultados similares fueron reportados en investigaciones donde se aplicó hierro-dextrano en diferentes dosis y el peso fue medido al destete. En este trabajo también se menciona que la tasa de crecimiento se vio afectada cuando el nivel de hemoglobina se redujo a $5 \mathrm{~g} / \mathrm{dl}^{21}$. Otros señalaron que el peso puede verse comprometido cuando los niveles de hemoglobina se mantienen por debajo de $8 \mathrm{~g} / \mathrm{dl}^{22}$.

Adicionalmente, la suplementación dietaria de hierro mejora el recuento total de células rojas, la tasa de hemoglobina y la concentración de Fe en plasma e hígado. No obstante, la suplementación indiscriminada de Fe (250 mg/kg de alimento) debe evitarse pues está asociada al incremento de la incidencia de diarrea y a la reducción de la tasa de crecimiento ${ }^{12}$. El aumento de peso corporal no es un indicador sensible de la deficiencia de Fe y las reducciones en la tasa de crecimiento ocurren solo en las etapas finales de la deficiencia de este micronutriente ${ }^{2}$.

Tras evaluar el efecto de 3 protocolos de administración de hierro en lechones, a través de la determinación de variables hematológicas se encontró que la aplicación de dos dosis de hierro-dextrano (100 mg/dl) a los 3 y 7 días de edad, fue más efectiva para prevenir la anemia, como lo reflejan los valores de hematíes, hemoglobina y hematocrito. El peso vivo del lechón no se vio favorecido por ninguno de los tratamientos aplicados.

\section{REFERENCIAS}

1. Almond G, Byers E, Seate J, Boyer P. 2017. Supplemental iron dextran injections: Influence on hemoglobin concentrations and piglet growth. $J$ Swine Health Prod 25 : 308-312.

2. Amine EK, Neff R, Hegsted DM. 1972. Biological estimation of available iron using chicks or rats. J Agr Food Chem 20: 246-251.

3. Bhattarai S, Nielsen JP. 2015. Association between hematological status at weaning and weight gain post-weaning in piglets. Livest Sci 182: 64-68.

4. Braude R, Chamberlain AG, Kotarbinska M, Mitchell KG. 1962. The metabolism of iron in piglets given labelled iron either orally or by injection. Br J Nutr 16: 427-449.

5. Calvo JJ, Allue JR. 1986. Plasma ferritin and other parameters related to iron metabolism in piglets. Comp Biochem Physiol 85: 471-476.

6. Carr J. 2014. Guia práctica para el manejo del ganado porcino, 1ra ed., Servet Ed., Zaragoza, p. 16.

7. Ducsay CA, Buhi WC, Bazer FW, Roberts RM, Combs GE. 1984. Role of uteroferrin in placental iron transport: effect of maternal iron treatment on fetal iron and utero- ferrin content and neonatal hemoglobin. J Anim Sci 59: 1303-1308.

8. Furugouri K, Miyata Y, Shijimaya K, Narasaki N. 1983. Developmental changes in serum ferritin of piglets. J Dairy Sci 67: 1256-1263.

9. Ishaya V, Ishaya M. 2012. Iron nutrition and anaemia in piglets: a review. $J$ Vet $A d v 2$ 2: 261-265.

10. Jackson P, Cockcroft P. 2009. Manual de medicina porci$n a$, lera ed., Editorial Inter-Médica, Buenos Aires, p. 230232

11. Jolliff JS, Mahan DC. 2011. Effect of injected and dietary iron in young pigs on blood hematology and postnatal pig growth performance. J Anim Sci 89: 4068-4080.

12. Lee SH et al. Effects of dietary iron levels on growth performance, hematological status, liver mineral concentration, fecal microflora, and diarrhea incidence in weanling pigs. Biol Trace Elem Res 126: 57-68.

13. Lewis AJ, Southern LL. 2000. Swine nutrition, 2nd ed., CRC press, New York. p. 251-254.

14. Li Y et al. 2018. Effect of different sources and levels of iron in the diet of sows on iron status in neonatal pigs. Anim Nutrit 4: 197-202.

15. Lipinski P et al. 2010. Benefits and risks of iron supplementation in anemic neonatal pigs. Br J Psychiatry 111: 1009-1010.

16. McDowell LR. 2003. Minerals in animal and human nutrition, 2nd. ed., p. 206-232, Elsevier Publisher, Florida, $660 \mathrm{p}$.

17. National Research Council. 2012. Nutrient requirements of swine, 11th ed., National Academic Press, Washington DC, p. 84-85.

18. Perri AM, Friendship RM, Harding JC, O'Sullivan TL. 2016. An investigation of iron deficiency and anemia in piglets and the effect of iron status at weaning on postweaning performance. $J$ Swine Health Prod 24: 10-20.

19. Peters JC, Mahan DC. 2008. Effects of neonatal iron status, iron injections at birth, and weaning in young pigs from sows fed either organic or inorganic trace minerals. $J$ Anim Sci 86: 2261-2269.

20. Pu Y et al. 2018. Iron promotes intestinal development in neonatal piglets. Nutrients 10: 1-11.

21. Ranjan R, Prasad CM, Singh SK. 2012. Effect of iron dextran injection on growth performance of crossbred and desi piglets under farm and village conditions. Vet World 5: 599-602.

22. Smith J, Moore K, Boyington D. 1983. Enzyme immunoassay for serum ferritin of pigs. Biochem Med 29: 293-297.

23. Starzynski RR et al. 2013. Iron supplementation in suckling piglets: How to correct iron deficiency anemia without affecting plasma hepcidin levels. PLOS ONE 8: e64022.

24. Svoboda M, Ficek R, Drábek J. 2008. Reticulocyte indices in the diagnosis of iron deficiency in suckling piglets. $B$ Vet I Pulawy 52: 125-130.

25. Svoboda M, Vaňhara J, Berliska J. 2017. Parenteral iron administration in suckling piglets, a review. Acta Vet Brno 86: 249-261

26. Szudzik M et al. 2018. Iron supplementation in suckling piglets: An ostensibly easy therapy of neonatal iron deficiency anemia. J Pharm 11: 128. 
27. Thorén K. 1984. Studies of serum-transferrin and some hematologic parameters in vitamin $\mathrm{E}$ and selenium deficient pigs. Ann Rech Vet 15: 387-394.

28. Valenzuela C, Antileo R, Lagos G, Pizarro F. 2015. El cerdo como modelo experimental para la nutrición de hierro. Rev Chil Nutr 42: 191-198.
29. Venn JA, McCance RA, Widdowson EM. 1947. Iron metabolism in piglet anaemia. J Comp Pathol 57: 314-325.

30. Ventrella D et al. 2017. The biomedical piglet: Establishing reference intervals for haematology and clinical chemistry parameters of two age groups with and without iron supplementation. BMC Vet Res 13: 1-8.

\section{Revista Veterinaria ingresa a SciELO}

Revista Veterinaria, publicación oficial de la Facultad de Ciencias Veterinarias de la Universidad Nacional del Nordeste (Corrientes, Argentina), ha logrado acceder al Núcleo Básico de Revistas Científicas Argentinas (Nivel 1), luego de calificar adecuadamente en el Centro Argentino de Información Científica y Tecnológica (CAICYT), según Resolución 2485/14 del Consejo Nacional de Investigaciones Científicas y Técnicas (CONICET).

Sobre un puntaje máximo de 33 se obtuvieron 32 puntos. Tal calificación constituye "una garantía de la excelencia de la publicación" (sic) y queda expedita la vía del Portal SciELO (Scientific Electronic Library Online) para los artículos publicados.

En tal calificación gravitó positivamente la circunstancia de haber aumentado el índice de impacto (Scimago-Elsevier) y haber disminuido las autocitaciones. También se tuvieron en cuenta aspectos como la amplia cobertura de la revista, la calidad científica del Comité Editorial, los criterios de evaluación de los artículos, el origen de los autores (locales 60\%, nacionales 13\%, extranjeros $24 \%$, en idioma inglés), el adecuado balance entre trabajos científicos originales y reseñas bibliográficas (ambos con alta calidad), así como el estricto cumplimiento de la periodicidad semestral y la favorable acogida por indizadores como Cab, J-Gate, Doaj, Ebsco, Gale Cengage, Infocyt, Latindex y Scopus.

Se consolida de esta manera la continuidad de "Revista Veterinaria", que en su acontecer registra más de 50 años de existencia en nuestra Facultad de Ciencias Veterinarias, entidad que en 2019 cumplió el $99^{\circ}$ aniversario de su fundación. 